\title{
Obstructive Hydronephrosis with Secondary Urosepsis
}

\author{
Shao-Lun Hsu ${ }^{1}$, Hsin-Yi Liu ${ }^{2}$, and Chia-Kwung Fan ${ }^{3}$ \\ ${ }^{1}$ Taipei Tzu Chi Hospital \\ ${ }^{2}$ Taipei Medical University Hospital \\ ${ }^{3}$ Taipei Medical University College of Medicine
}

September 30, 2021

\begin{abstract}
Obstructive uropathy is one of the leading causes of urosepsis. In the patients with severe hydronephrosis, underlying sepsis and renal dysfunction should be treated with caution. Each additional hour of delay in antibiotics administration was found to lower the survival rate.
\end{abstract}

\section{Obstructive Hydronephrosis with Secondary Urosepsis}

Shao-Lun Hsu ${ }^{1,2}$, Hsin-Yi Liu ${ }^{3}$, Chia-Kwung Fan ${ }^{2}$

${ }^{1}$ Medical Education Department, Taipei Tzuchi University Hospital, New Taipei City, Taiwan

2 Department of Molecular Parasitology and Tropical Diseases, School of Medicine, College of Medicine, Taipei Medical University, Taipei, Taiwan

${ }^{3}$ Division of Infectious Diseases, Department of Internal Medicine, Taipei Medical University Hospital, Taipei, Taiwan

A 63-year-old male patient presented to the emergency department due to fever, dyspnea, and a one-month history of dyspepsia and body weight loss of 10kg. On examination, decreased blood pressure, costovertebral angle tenderness with left abdominal palpable mass was notable. Laboratory studies revealed leukocytosis and renal dysfunction. Urine analysis showed pyuria, hematuria and proteinuria. There were no reports of dysuria or a decrease in urine output. CT revealed a gross-dilated mass lesion with homogeneous contents.

\section{Quiz Question: What is your diagnosis?}

Computed tomography showed a classic balloon-like shape ("balloon on a string" sign $^{1}$ ) in grade IV hydronephrosis with gross dilatation of the renal pelvis and calyces. The indistinguishable corticomedullary differentiation, membrane-like residual cortex (parenchymal rim), indicates renal atrophy ${ }^{2}$. In the present case, the very small caliber of the left ureter suggested ureteropelvic junction stricture. The diagnosis was urosepsis secondary to obstructive hydronephrosis. Hydronephrosis is one of the leading causes of severe urinary tract infection and renal dysfunction which should beware of ongoing sepsis. Escherichia coli was isolated from both urine culture and blood culture. After emergent percutaneous nephrostomy and antibiotic treatment by cefmetazole, he was discharged two weeks later with recovered renal function.

\section{Reference}

Dyer RB, Chen MY, Zagoria RJ. Classic Signs in Uroradiology. 2004. Radiographics 24(1): S247-80.

Lee SH, Lee JW, Park JT, Kim HI, Im HW, Kim YS, Kim SJ. Secondary polycythemia caused by giant hydronephrosis due to ureteropelvic junction obstruction. 2007. Korean J Urol 48(9):984-985. 


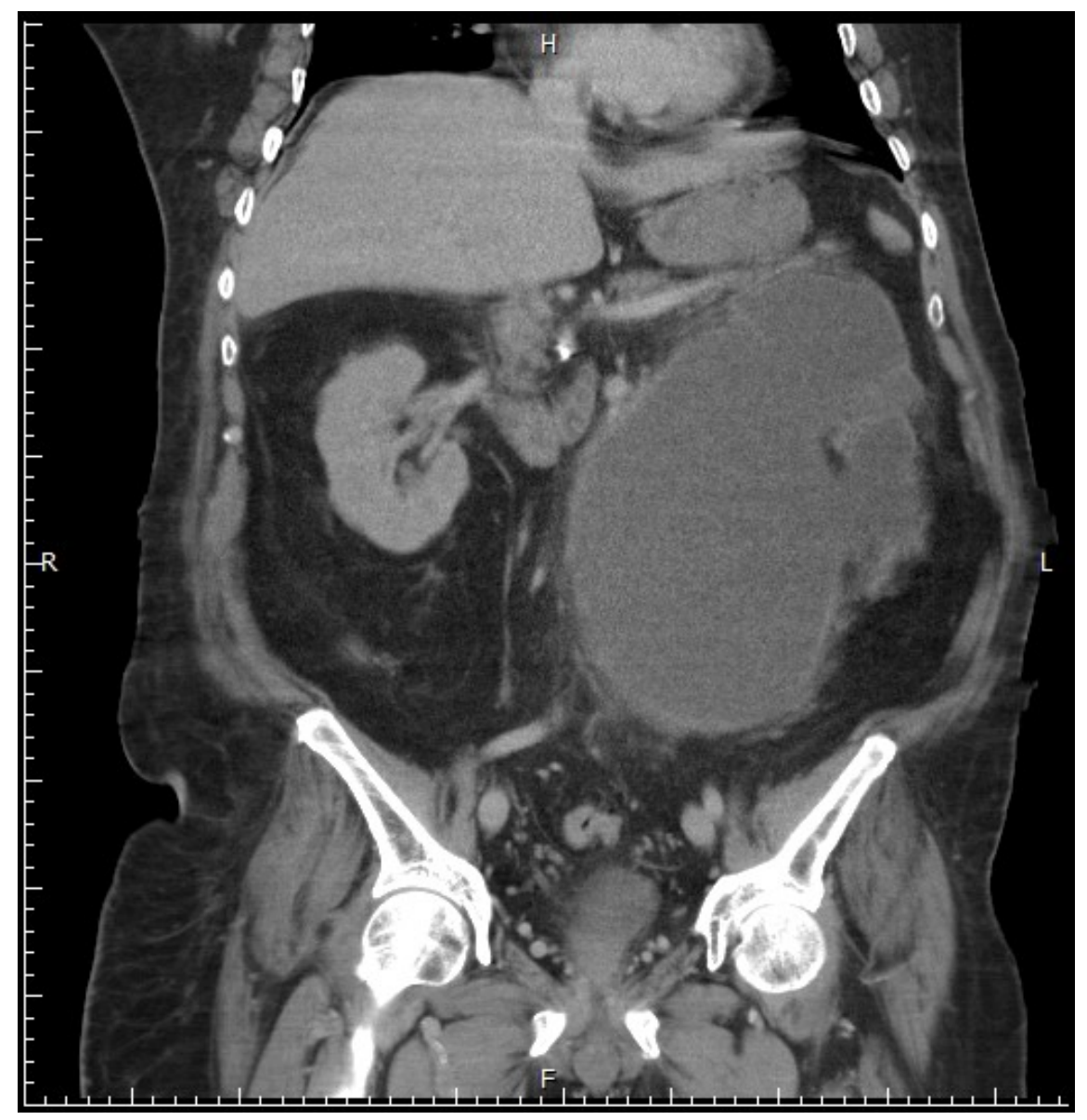

